\title{
Correlación entre corioamnionitis histológica y clínica en pacientes con ruptura prematura de membranas mayor de 12 horas
}

\author{
Javier U. Ortiz, MD.*; Mario A, Rebolledo, MD.**; Ricardo Alvarado, MD.***
}

\section{RESUMEN}

OBJETIVOS: Los objetivos de este estudio fueron: establecer la frecuencia de corioamnionitis histológica en pacientes con ruptura prematura de membranas con y sin evidencia clínica de corioamnionitis, correlacionar los factores de riesgo para desarrollar corioamnionitis y establecer el tipo histológico más frecuente de corioamnionitis en la población estudiada

MATERIALES Y METODOS: Se evaluaron los signos clínicos, histológicos y factores de riesgo para corioamnionitis y las complicaciones maternas y fetales en pacientes con embarazo $\geq 24$ semanas, diagnóstico de ruptura prematura de membranas y tiempo $\geq 12$ horas entre la ruptura prematura de membranas y el parto.

RESULTADOS: De 55 pacientes con ruptura prematura de membranas, $30(54,5 \%)$ no tuvieron corioamnionitis, 18 (32,7\%) tuvieron corioamnionitis histológica únicamente y $7(12,7 \%)$ tuvieron corioamnionitis histológica y clínica. El estadio II (corionitis) fue el más frecuente $(\mathbf{4 8} \%)$. La aparición de algún signo clínico favorece el parto por vía vaginal $(p=0,04)$. Los factores de riesgo no se correlacionan con la aparición de corioamnionitis $(p=N S)$. Se presentó una paciente con endometritis postcesárea y un recién nacido con neumonía in útero.

CONCLUSIONES: La corioamnionitis histológica es más frecuente que la corioamnionitis clínica. Los signos clínicos se correlacionan siempre con la corioamnionitis histológica.

PALABRAS CLAVES: Ruptura prematura de membranas, corioamnionitis histològica, corioamnionitis clínica.

\section{SUMMARY}

OBJECTIVES: The objetives of the study were to establish the frecuency of histologic chorioamnionitis in patients with premature rupture of membranes with and without clinical evidence of amnionitis, to determine the risk factors in the development of chorioamnionitis and to determine the most frecuent histologic subtype of chorioamnionitis in the population studied.

MATERIALS AND METHODS: We evaluated the clinical and histologic signs of chorioamnionitis as weII as the risk factors for chorioamnionitis and the maternal and fetal complications in patients with gestational age $\geq 24$ weeks who had premature rupture of membranes and a period $\geq 12$ hours between the rupture of the membranes and the delivery.

RESULTS: Of the 55 pacients with premature rupture of membranes participating in the study, 30 (54,5\%) did not have chorioamnionitis. $18(37,7 \%)$ had only histologic evidence of chorioamnionitis and $7(12,7 \%)$ had clinical and histologic evidence of chorioamnionitis. The stage II (corionitis) was the most frecuent histologic subtype $(\mathbf{4 8 \%})$. The presence of some clinical sign favoured vaginal delivery $(p=0,04)$. The presence of risk factors were not related to the presentation of chorioamnionitis $(p=N S)$. We had one patient with postpartum endometritis and one newborn with intrauterine pneumonia.

CONCLUSIONS: Histologic chorioamnionitis is more frecuent than clinical chorioamnionitis. Clinical signs are always related with histologic evidence of chorioamnionitis.

KEY WORDS: Premature rupture of membranes, histologic chorioamnionitis, clinical chorioamnionitis.

\section{Introducción}

Durante la gestación, el moco cervical y las membranas ovulares forman una barrera importante que separa al feto y al líquido amniótico estériles del canal vaginal

Residente III año GO HILVdeS.

** Instructor Unidad de Medicina Materno-Fetal HILVdeS.

*** Jefe Departamento de Patología HILVdeS. cargado de bacterias, por lo que casi nunca se observan gérmenes en el líquido amniótico antes del parto (1-2).

La pérdida de la integridad de las membranas corioamnióticas antes del inicio del trabajo de parto, recibe el nombre de ruptura prematura de membranas (RPM) (3).

$\mathrm{Su}$ incidencia varía del 3 al $18 \%$ (4). Esta amplia diferencia puede corresponder a variaciones en la definición y en la población estudiada. 
Aproximadamente el 8-10\% de pacientes con embarazo a término la presentan. El $25 \%$ de los casos de RPM ocurre antes del término y es la responsable del $30 \%$ de los partos pretérmino (5).

La RPM tiene como complicaciones: infección materna (corioamnionitis), infección fetal, trabajo de parto pretérmino, parto pretérmino, oligoamnios e hipoxia y asfixia fetal secundaria a compresión del cordón umbilical.

Se define corioamnionitis como la infección de las membranas fetales por bacterias o mycoplasmas (5). Su incidencia es del 0,5 - 10\% de todos los embarazos (6).

Se han establecido 3 vías de infección: 1) ascendente: es la más frecuente, ocurre en los casos de RPM y fue la que se estudió en el presente trabajo. 2) Hematógena: producida por listeria monocytogenes, estreptococo del grupo B y campylobacter. 3) Procedimientos obstétricos invasivos: amniocéntesis $(1: 1000)$, transfusión intrauterina (5\%), cerclaje cervical (1-2\%), fetoscopia y biopsia de las vellosidades coriales (7).

Una vez ocurre el ingreso del microorganismo, los neutrófilos maternos de la sangre que baña al espacio intervelloso, son atraídos para fijarse inicialmente en la lámina basal de la placa coriónica (estadio I: subcorionitis). A continuación migran hacia el interior de la placa (estadio II: corionitis). Por último alcanza la membrana basal del amnios (estadio III: corioamnionitis) (5). Normalmente no pasan a través de esta membrana basal hacia el líquido amniótico ya que no poseen una enzima que rompa el colágeno tipo $\mathrm{V}$, constituyente importante de dicha membrana (8).

Los neutrófilos fetales de los vasos umbilicales son atraídos con frecuencia por sustancias quimiotácticas del líquido amniótico, lo que produce una migración hacia la cavidad amniótica. Con menor frecuencia, los neutrófilos del líquido amniótico migran hacia el cordón umbilical. En la evolución de la infección, esta migración de las células inflamatorias ocurre inicialmente en la vena y posteriormente en las arterias umbilicales. Este proceso recibe el nombre de funisitis (5).

Los objetivos de este estudio fueron establecer la frecuencia de corioamnionitis histológica en pacientes con ruptura prematura de membranas con y sin evidencia clínica de corioamnionitis, correlacionar los factores de riesgo para desarrollar corioamnionitis y establecer el tipo histológico más frecuente de corioamnionitis en la población estudiada.

\section{Materiales y Métodos}

Se realizó un estudio de casos y controles anidado en una cohorte de pacientes con diagnóstico de RPM, con un período de tiempo mayor o igual a 12 horas entre la RPM y el parto, y con embarazos mayores o iguales a 24 semanas que consultaron al servicio de urgencias del Departamento de Ginecología y Obstetricia del Hospital Infantil Universitario "Lorencita Villegas de Santos", institución de maternidad de tercer nivel, entre junio de 1996 y julio de 1997.

Se excluyeron aquellas que venían recibiendo antibiótico al momento del ingreso.
En la historia clínica se consignó información acerca de relaciones sexuales, flujo vaginal y sintomatología de infección de vías urinarias (IVU) como disuria, polaquiuria, urgencia y tenesmo vesical presentes en las últimas 72 horas. Además dentro de los antecedentes se anotó la presencia de enfermedad inmunosupresora (diabetes mellitus, colagenopatías, síndromes de inmunodeficiencia y otras) y la administración de medicamentos inmunosupresores (corticoides, antineoplásicos y otros), todos estos conocidos factores de riesgo para desarrollar corioamnionitis (9).

Durante el examen físico se realizó cervicovaginoscopia con espéculo estéril y se hizo diagnóstico de RPM al evidenciar líquido amniótico en el fondo de saco posterior. Se practicó además un tacto vaginal (TV) para determinar las características del cuello.

Luego las pacientes fueron hospitalizadas en sala de partos, y manejadas de la siguiente manera: restricción en el número de tactos vaginales, compresa estéril en periné, medición de temperatura axilar y pulso materno cada 2 horas y auscultación de la frecuencia cardíaca fetal cada 20 minutos. También se solicitó un cuadro hemático y un parcial de orina con sonda.

Se hizo diagnóstico de corioamnionitis clínica si se presentaba uno de los siguientes signos: temperatura materna ${ }^{3} 37,8^{\circ} \mathrm{C}$, taquicardia materna $\left({ }^{3} 100 \mathrm{x}\right.$ '), taquicardia fetal (>160 x'), olor fétido del líquido amniótico o leucocitosis materna $(>12.000)$ con cambios tóxicos en el diferencial (neutrofilia o cayademia) (10).

A todas las pacientes con diagnóstico de corioamnionitis, se les inició antibióticoterapia (Penicilina cristalina 5'000.000 un IV c/4 horas o Ampicilina 1 gr. IV c/6 horas) y, posterior al parto (vaginal o por cesárea), se agregó Gentamicina $80 \mathrm{mg} \mathrm{lV}$ c/8 horas.

Todas las pacientes que terminaron su parto por cesárea que no tuvieron signos clínicos de corioamnionitis, recibieron dosis profilácticas de antibiótico (Ampicilina o Cefalotina).

Las placentas fueron depositadas en bolsas estériles con formaldehido al $10 \%$ y enviadas para estudio anatomopatológico dentro de las 24 horas siguientes a la recolección. A las placentas se les realizaron cuatro cortes con micrótomo, así: uno en el cordón umbilical a $2 \mathrm{~cm}$ de su inserción en la placenta, uno en las membranas fetales del plato placentario en una zona periférica a la inserción del cordón umbilical y dos desde el borde de inserción hasta el borde libre de las membranas fetales en las hojas anterior y posterior.

Los cortes fueron incluidos en parafina y coloreados con Hematoxilina-Eosina.

La lectura de las placas se realizó con microscopio binocular a $40 \mathrm{x}-100 \mathrm{x}-450 \mathrm{x}$ por una sola persona. La disposición periférica de los PMN en la vena y/o arterias umbilicales se llamó funisitis (5).

Se revisaron las historias clínicas materna (edad, edad gestacional, perfil obstétrico, signos clínicos e histológicos de corioamnionitis, factores de riesgo, tipo del parto, días de hospitalización y complicaciones) y del recién nacido (edad, indicación de hospitalización, complicaciones, días de hospitalización), recopilando la información en un formato destinado para tal fin. Se hizo el análisis estadístico utilizando el programa EPI INFO 6,01. Una $p<0,05$ 
fue considerada estadísticamente significativa. Se estimó la asociación entre los factores de riesgo y la RPM por medio de la prueba chi cuadrado y/o el OR.

\section{Resultados}

Se estudiaron 55 pacientes entre el 1-VI/96 y el 31VII/97. Las características demográficas de la población estudiada se muestran en la tabla 1 .

De las 55 pacientes estudiadas, $30(54,5 \%)$ no tuvieron corioamnionitis, $18(32,7 \%)$ tuvieron corioamnionitis histológica únicamente y $7(12 ; 7 \%)$ tuvieron corioamnionitis histológica y Clínica. De las 25 pacientes con corioamnionitis histològica, $9(36 \%)$ se clasificaron como subcorionitis (estadio I), 12 (48\%) como corionitis (estadio II) y $4(16 \%)$ como corioamnionitis (estadio III). Se presentó funisitis en 7 pacientes: 2 (8\%) concomitantemente con estadio I, 4 (16\%) con estadio II (en 2 aparecieron signos clínicos) y 1 (4\%) con estadio III.

Los signos clínicos que se presentaron en las pacientes con corioamnionitis fueron: olor fétido del líquido amniótico en $4(57,1 \%)$, leucocitosis en $3(42,8 \%)$, taquicardia materna en $2(28,6 \%)$, fiebre en $1(14,3 \%)$ y taquicardia fetal en $1(14,3 \%)$.

Se encontró una asociación estadísticamente significativa entre la presencia de signos clínicos y la evidencia de corioamnionitis histológica $(\mathrm{p}=0,02)$.

Los factores de riesgo presentes en las 25 pacientes con corioamnionitis fueron: relaciones sexuales en 5 (20\%), flujo vaginal en $2(8 \%)$, infección urinaria en 1 (4\%), tiempo promedio RPM-Parto: $22,8 \pm 9,1$ y mediana de TV: 5 ; y en las 30 pacientes sin corioamnionitis fueron: relaciones sexuales en $2(6,6 \%)$, flujo vaginal en $5(16,6 \%)$, IVU en $1(3,3 \%)$, tiempo promedio RPMParto: $19,8 \pm 8,5$ y mediana de T.V.: 4 .

Ni la edad materna, ni la edad gestacional, ni los factores de riesgo se correlacionan con la aparición de corioamnionitis $(p=$ NS) (Tabla 2).

Ninguna paciente presentaba enfermedad inmunosupresora o requería medicación inmunosupresora.

Las pacientes con corioamnionitis clínica tuvieron un número de días de hospitalización mayor $(3,7 \pm 1,6)$, que aquellas que no presentaron signos clínicos $(2,3 \pm$ $1,1)(\mathrm{p}=0,02)$.

La presencia de corioamnionitis histológica (incluyendo las 7 con corioamnionitis clínica) no intervino en la vía del parto y aún cuando no fue significativo hay una tendencia a terminar en cesárea ( $\mathrm{p}=0,06$ OR 3,1 IC 0,812,5) (Tabla 3).

La aparición de algún signo clínico se constituye en un factor protector para parto por vía abdominal ( $\mathrm{p}=0,04$ OR 0,1 IC $0,02-0,9$ ).

De las 55 pacientes, a $17(30,9 \%)$ se les realizó cesárea. De éstas, $11(64,7 \%)$ tuvieron corioamnionitis $(6-54,5 \%$ -histológica únicamente y 5 - 45,5\% - histológica y clínica) y $6(35,3 \%)$ no tuvieron corioamnionitis.

Sus indicaciones fueron: distocia dinámica en $7(41,1 \%)$, placenta marginal en $2(11,8 \%)$, presentación podálica en 2 $(11,8 \%)$, insuficiencia placentaria en $1(5,9 \%)$, iterativa I en $2(11,8 \%)$, desproporción cefalo-pélvica (DCP) en 2(11,8\%) y presentación de cara en $1(5,9 \%)$ (Tabla 4$)$.
Tabla 1

CARACTERISTICAS DEMOGRAFICAS

\begin{tabular}{|lc|}
\hline Edad materna & $25,1 \pm 5,7(15-39)$ \\
Edad gestacional & $38,5 \pm 2,1(29-41)$ \\
Gravidez & $1,7 \pm 1,0(1-5)$ \\
Paridad & $0,6 \pm 0,9(0-3)$ \\
Tiempo RPM - Parto & $21,1 \pm 8,8(12-54)$ \\
\hline
\end{tabular}

$\mathrm{n}=55$

Tabla 2

ANALISIS UNIVARIADO DE FACTORES DE RIESGO, VIA DEL PARTO Y DIAS DE HOSPITALIZACION

\begin{tabular}{|lccc|}
\hline & $\begin{array}{c}\text { Corioam- } \\
\text { nionitis } \\
(\mathbf{n}=\mathbf{2 5})\end{array}$ & $\begin{array}{c}\text { No } \\
\text { Corioam- } \\
\text { nionitis } \\
(\mathbf{n = 3 0})\end{array}$ & $\mathbf{p}$ \\
\hline Edad materna & $24,9 \pm 5,2$ & $25,2 \pm 6,2$ & NS \\
Edad gestacional & $38,1 \pm 2,8$ & $38,9 \pm 1,2$ & NS \\
Tiempo RPM-Parto & $22,8 \pm 9,1$ & $19,8 \pm 8,5$ & NS \\
Flujo vaginal & 2 & 5 & NS \\
Relaciones sexuales & 5 & 2 & NS \\
Infección vías & & 1 & NS \\
urinarias & 1 & 4 & NS \\
Tactos vaginales & 5 & 6 & NS \\
Parto por cesárea & 11 & 24 & NS \\
Parto vaginal & 14 & & \\
Días de hospita- & & & \\
lización materna & $2,8 \pm 1,3$ & $2,3 \pm 1,1$ & NS \\
\hline
\end{tabular}
Tabla 3
ASOCIACION ENTRE CORIOAMNIONITIS Y VIA
DEL PARTO

\begin{tabular}{|l|ccc|}
\hline \multirow{2}{*}{ Corioamnionitis } & \multicolumn{3}{|c|}{ Tipo parto } \\
\cline { 2 - 4 } & Cesárea & Vaginal & Total \\
\hline $\mathrm{Si}$ & 11 & 14 & 25 \\
No & 6 & 24 & 30 \\
Total & 17 & 38 & 55 \\
\hline
\end{tabular}

$\mathrm{p}=\mathrm{NS}$ OR 3,1 IC $0,8-12,5$

Tabla 4

INDICACIONES DE CESAREA

\begin{tabular}{|l|ccc|}
\hline \multirow{2}{*}{ Indicación } & \multicolumn{3}{c|}{ Corioamnionitis } \\
\cline { 2 - 4 } & Si & No & Total \\
\hline Distocia Dinámica & 5 & 2 & 7 \\
Placenta Marginal & 2 & 0 & 2 \\
Podálico & 1 & 1 & 2 \\
Insuficiencia placentaria & 0 & 1 & 1 \\
Iterativa I & 2 & 0 & 2 \\
DCP & 0 & 2 & 2 \\
Presentación de cara & 1 & 0 & 1 \\
Total & 11 & 6 & 17 \\
\hline
\end{tabular}


Las pacientes con cesárea se quedaron más días hospitalizadas $(3,0 \pm 1,0)$ que las que tuvieron el parto por vía vaginal $(2,3 \pm 1,3)(\mathrm{p}=0,009)$.

Se hospitalizaron 10 recién nacidos: 7 (70\%) hijos de madres con corioamnionitis ( 3 con corioamnionitis clínica) y $3(30 \%)$ hijos de madres sin corioamnionitis.

Las indicaciones de hospitalización fueron: ictericia $\mathrm{ABO}$ en $2(20 \%)$, ictericia fisiológica en $2(20 \%)$, observación por infección materna en $2(20 \%)$, prematurez en $1(10 \%)$, síndrome de dificultad respiratoria (SDR) en 2 (20\%) y broncoaspiración de meconio en $1(10 \%)$.

Los R.N. pretérmino hijos de madre con o sin corioamnionitis se quedaron más días hospitalizados $(21,7$ $\pm 2,1)$ que los que estaban a término $(3,0 \pm 0,6)(\mathrm{p}=0,01)$.

No hubo correlación entre la presencia de corioamnionitis y la edad gestacional al nacimiento, la indicación y el número de días de hospitalización del recién nacido $(\mathrm{p}=\mathrm{Ns})$.

\section{Discusión}

El presente estudio mostró una incidencia de $45.5 \%$ para corioamnionitis histológica y de $12.7 \%$ para la clínica.

Otros estudios refieren que la incidencia de corioamnionitis histológica $(15-70 \%)$ es mayor cuando se compara con la corioamnionitis clínica (5 - 25\%), por lo que nuestros hallazgos están de acuerdo con lo reportado en la literatura (11-12).

En nuestro trabajo el tipo histológico más frecuente fue la corionitis (estadio II) que se presentó en 12 pacientes $(48 \%)$.

La aparición de funisitis (7 pacientes) y de signos clínicos (7 pacientes) no se correlacionan con el mayor grado histológico encontrado. Esto difiere del trabajo de Dong et al en donde encontraron una asociación importante entre la mayor infiltración de las membranas fetales y la aparición de signos clínicos y funisitis (80\%). Esto podría explicarse porque los criterios histopatológicos usados por ellos se basaban en el número de PMN presentes por campo de gran aumento $(1+: 1-3,2+: 4-15$, $3+:>15)$ y porque el corte del cordón lo hacían en la zona de inserción a la placenta (13).

Los factores de riesgo tales como tiempo entre la RPM y el parto, número de tactos vaginales, flujo vaginal, relaciones sexuales e IVU no intervinieron en una mayor aparición de corioamnionitis. Esto podría explicarse por el pequeño número de pacientes que los presentaban y por el tiempo entre la RPM y el parto en la población estudiada.

Soper et al, en su estudio de 408 pacientes, encontraron una correlación significativa entre los factores de riesgo y corioamnionitis. Sin embargo, en ese estudio, todas las pacientes eran indigentes, el tiempo entre la RPM y el parto era de $24,3 \pm 18,1$ y se utilizó monitorización interna en el 72,5\% de las pacientes (14).

Silver et al demostraron una importante asociación entre la corioamnionitis y el flujo vaginal bacteriano. En este estudio $22(69 \%)$ de las 32 pacientes con corioamnionitis, presentaban flujo vaginal (15).
En las pacientes con corioamnionitis clínica se reserva el parto por cesárea para aquellas con una indicación obstétrica establecida ya que aumenta la morbilidad materna. Sin embargo, la tasa de cesáreas se incrementa en un $35-40 \%$, principalmente como resultado de distocia dinámica (7).

Encontramos que una paciente con corioamnionitis histológica y/o clínica tiene una probabilidad mayor de terminar su parto por vía abdominal ( $\mathrm{p}=0,06$ OR 3,1 IC $0,8-12,5$ ). Quizá con un número mayor de pacientes se hubiera encontrado significancia estadística. La causa más frecuente de cesárea en pacientes con corioamnionitis fue la distocia dinámica $(45,5 \% \mathrm{p}=\mathrm{NS})$.

Gilstrap et al encontraron que las complicaciones maternas que se presentaron en pacientes con corioamnionitis, estuvieron relacionadas principalmente con la infección postparto, siendo una de las más frecuentes, la endometritis postparto (16).

Sólo una paciente con corioamnionitis histológica (estadío II) y clínica a quien se le realizó cesárea por presentación podálica, presentó endometritis postparto.

No hubo complicaciones infecciosas postoperatorias en ninguna de las 6 pacientes que presentaron corioamnionitis histológica únicamente y que terminaron su parto por vía abdominal. Quizá estas pacientes no requieran antibióticoterapia o la sola dosis profiláctica sea suficiente para prevenir la infección.

Dentro de la morbilidad fetal y del recién nacido, se presentan múltiples entidades. El más frecuente de estos hallazgos es la neumonía. También pueden presentarse otitis, sinusitis, meningitis, gastroenteritis y septicemia. $(3,17)$.

Un recién nacido de 36 semanas, hijo de madre con corionitis (estadío II) y sin signos clínicos de corioamnionitis, presentó neumonía in útero y enterocolitis IB dándose de alta a los 20 días de hospitalización. Otro recién nacido de 29 semanas, hijo de madre con subcorionitis (estadio I) únicamente, fue hospitalizado por enfermedad de membrana hialina. Durante su evolución presentó neumonía e infección de vías urinarias falleciendo al $24^{\circ}$ día. Probablemente este único caso de mortalidad neonatal esté dado más por complicación de la prematurez que por la infección.

Este estudio demostró una mayor incidencia de corioamnionitis histológica sobre la clínica y la relación directa entre corioamnionitis clínica e histológica.

No encontramos una significancia estadística entre los factores de riesgo y la presencia de corioamnionitis, tal vez por el tamaño de la muestra o porque no estamos interrogando los verdaderos factores de riesgo que intervienen en el desarrollo de la corioamnionitis en nuestra población.

Finalmente, ć́eemos que sería ideal encontrar una prueba diagnóstica prenatal de corioamnionitis histològica para iniciar un tratamiento precoz, disminuyendo así la morbi-mortalidad materno-fetal.

Agradecemos la valiosa colaboración de Jaime E. Ruiz, MD, y Claudia Ruetz. 


\section{BIBLIOGRAFIA}

1. Garite TJ. Premature rupture of the membranes. En: Creasy RK, Resnik R, eds. Maternal-fetal medicine: principles and practice. Philadelphia: WB Saunders; 1994; 625-38.

2. Gibbs RS. Corioamniotis y patología relacionada con la monitorización intrauterina. En: Monif GR, ed. Enfermedades infecciosas en obstetricia y ginecología. Barcelona: Salvat; 1985; 353-63.

3. Sánchez F. Ruptura prematura de membranas. En: Sánchez F, ed Alto riesgo obstétrico. Santafé de Bogotá: Universidad Nacional de Colombia; 1992; 172-5.

4. Arias F. Rotura prematura de membranas. En: Añas F, ed Guía práctica para el embarazo y el parto de alto riesgo. Madrid: Mosby/ Doyma; 1994; 101-14.

5. Naeye RL. Placenta y membranas. En: Sanford I, Tyrell D, Weller T, eds. Infecciones obstétricas y perinatales. Madrid: Mosby/Doyma; 1994; 101-14.

6. Gibbs RS. Obstetric factors associated with infections of the fetus and newborn infant. En: Remington JS, Klein JO, eds. Infectious diseases of the fetus and newborn infant. Philadelphia: WB Saunders; 1995; 1241-63.

7. Gibbs RS, Duff P. Progress in pathogenesis and management of clinical intraamniotic infection. Am J Obstet Gynecol 1991; 164: 1317-26.

8. Azzarelli B, Lafaza J. Amniotic basement membrane: a barrier to neutrophil invasion. Am J Obstet Gynecol 1987; 156: 1130-6.

9. Newton ER, Prihoda TJ, Gibbs RS. Logistic regression analysis of risk factors for intra-amniotic infection. Obstet Gynecol 1989; 73: 571-5.
10. Newton ER, Piper J, Pears W. Bacterial vaginosis and intraamniotic infection. Am J Obstet Gynecol 1997; 176: 672-7.

11. Gibbs RS, Romero R, Hillier SL. et al. A review of premature birth and subclinical infection. Am J Obstet Gynecol/1992; 166 1515-28.

12. Hillier SL, Martius J, Krohn M. et al. A case-control study of chorioamnionic infection and histologic chorioamnionitis in prematurity. N Engl J Med 1988; 319: 972-8.

13. Dong Y, Clair PJ, Ramzy I. et al. A microbiologic and clinical study of placental inflammation at term. Obstet Gynecol 1987; 70: 175-82.

14. Soper DE, Mayhall G, Dalton H. Risk factors for intraamniotic infection: a prospective epidemiologic study. Am J Obstet Gynecol 1989; 161: 562-8

15. Silver HM, Sperling RS, Clair PJ, et al. Evidence relating bacterial vaginosis to intraamniotic infection. Am J Obstet Gynecol 1989; 161 : $808-12$.

16. Gilstrap LC, Leveno KJ, Cox SM. Intrapartum treatment of acute chorioamnionitis: impact on neonatal sepsis. Am J Obstet Gynecol 1988; 159: 579-83.

17. Yoon BH, Romero R, Kim CJ. et al. Amniotic fluid interleukin-6: a sensitive test for antenatal diagnosis of acute inflammatory lesions of preterm placenta and prediction of perinatal morbidity. Am J Obstet Gynecol 1995; 172: 960-70. 